\title{
Educación Steam: entrada a la sociedad del conocimiento
}

\section{Steam education: entrance to the knowledge society}

Juan Patricio Santillán Aguirre. ${ }^{1}$, Valeria del Carmen Cadena Vaca. ${ }^{2}$ \& Miguel Cadena Vaca. ${ }^{3}$

\section{Recibido: 12-07-2019 / Revisado: 21-07-2019 /Aceptado: 14-08-2019/ Publicado: 10-09-2019}

\begin{abstract}
.
DOI: https://doi.org/10.33262/cienciadigital.v3i3.4..847

This article is a preview of an ongoing research, the purpose of which is to generate a theoretical construct about STEAM education. It is located in the interpretive paradigm under the approach of hermeneutics. The materials and method applied is a documentary research whose subject matter was the subject of four doctoral research carried out in the period from 2016-2017. The state of the art made it possible to determine a greater orientation of the steam education approach to the traditional model of teaching.
\end{abstract}

Keywords: Education, steam, knowledge society, focus, teaching.

\section{Resumen}

El presente artículo es un adelanto de una investigación en curso, cuyo propósito es generar un constructo teórico acerca de la educación STEAM. Se ubica en el paradigma interpretativo bajo el enfoque de la hermenéutica. Los materiales y el método aplicado es una investigación documental cuyo objeto de estudio lo constituyeron cuatro investigaciones doctorales realizadas en el período comprendido entre los años 20162017. El estado del arte permitió determinar una mayor orientación del enfoque de la educación STEAM en relación con el modelo tradicional de enseñanza.

Palabras clave: Educación, steam, sociedad del conocimiento, enfoque, enseñanza.

\section{Introducción.}

La base teórica sustantiva que identifica la caracterización del fenómeno de estudio en el involucramiento conceptual básico y contextual, que se presentan en el estado del arte respecto a los acontecimientos científicos que se significan como punto de sustentación percibido en la problematización investigativa, está vinculada a los razonamientos de diferentes autores y teorías que se interceptan según Rodríguez (2005) al describir el referencial teórico constitutivo de los elementos asociados, los cuales conforman una parte orgánica de la actividad indagatoria. De allí la importancia de su ampliación para la comprensión de las partes al todo y del todo a la integración de los puntos específicos preliminares del conocimiento en torno al apoyo de las

\footnotetext{
${ }^{1}$ Escuela Superior Politécnica de Chimborazo, Chimborazo, Ecuador, juan.santillan @espoch.edu.ec

${ }^{2}$ Unidad Educativa Jesuita San Felipe Neri, UESFN - Ecuador, vdcadena@sfelipeneri.edu.ec

${ }^{3}$ Escuela Superior Politécnica de Chimborazo, Chimborazo, Ecuador, miguelcadenavaca@yahoo.es
} 
propiedades que condicionan la mirada integral al objeto de estudio enriquecido en su máximo nivel conceptual.

Desde este punto de vista, este apartado de la investigación comprende el recorrido por estudios previos relacionados con el fenómeno y los fundamentos teóricos. He de resaltar que la metodología STEAM (Ciencia, Tecnología, Ingeniería, Artes y Matemáticas), según Jho, Hong \& Song (2016) conlleva a la apertura mental del docente y auto-innovación institucional en la significancia de actuaciones conjunta de los actores sociales al mantener un sistema de relación recíproca e intercambio continuo de roles como compromiso mutuo, de carácter colaborativo, creación de materiales educativos digitales y dedicación innovadora de estrategias de aprendizaje como repertorio compartido.

\section{Desarrollo}

\section{Recorrido por Estudios Previos Relacionados con el Fenómeno}

Diversidad de estudios doctorales se han presentado acerca de este fenómeno de la metodología STEAM implícita en las habilidades y capacidades para integrar áreas disciplinares al ir preparando al docente con esta metodología activa en el ejercicio profesional para la Sociedad del Conocimiento a través de la Educación STEAM. En este sentido, aunque el modelo STEM es diferente a la metodología STEAM, coincide en la integración interdisciplinar. De forma aclaratoria, se señala que el STEM, es el acrónimo bajo el cual se engloban las siglas Science, Technology, Engineering, Mathematics, actualmente conocida como STEAM, donde se le añade la letra A, referida a las Artes, lo cual da cabida a áreas del conocimiento relacionado con el arte y el diseño.

Al hacer esta aclaratoria, presento el estudio de Yildirim (2016), titulado: “An Examination of the Effects of Science, Technology, Engineering, Mathematics (STEM) Applications and Mastery Learning Integrated Into The 7th Grade Science Course" presentada en Gazi University, Ankara, cuyo propósito fue trazar un resumen de los estudios de investigación empírica realizados en la educación STEM focalizado en el logro del estudiante, además de su creatividad y habilidades de resolución de problemas, actitudes e intereses hacia los temas STEM. Se trató de una investigación documental, donde hubo la selección inicial de varios artículos, documentos y disertaciones sobre educación STEM, eligiendo treinta y tres (33) estudios, de acuerdo con el encuadre de interés investigativo. Los estudios seleccionados se sometieron al análisis y síntesis en línea con las etapas de la meta-síntesis, con el fin de especificar y presentar hallazgos en los temas encontrados. Allí se incluyeron los estudios alineados a los principios de educación STEM y las investigaciones de comparación internacional, como PISA/TIMSS, dado el impacto positivo en la educación STEM desde el rendimiento de los estudiantes en la escuela y en sus actitudes hacia las disciplinas STEM. Hubo evidencias que apoyaron las intervenciones que siguen a este tipo de metodología educativa. Los principios refuerzan la resolución de problemas y la creatividad de los estudiantes. 
Concluyó que la educación STEM incluye diferentes disciplinas en varias materias, por lo tanto, uno de los roles más importantes de esta metodología es que asegura que los docentes estén investigando, cuestionando y criticando a los individuos, lo cual ayuda a su propio desarrollo profesional como investigadores. Recomendó capacitar este dominio del conocimiento pedagógico apoyando al docente en instituciones internacionales para asimilar la educación STEM utilizando sus propios medios y del gobierno, con el fin de contribuir a su desarrollo y especificidad en esta área.

El aporte de este estudio previo a la investigación actual, concuerda en las expresiones reconocidas de los beneficios del efecto STEM en la educación para alcanzar el éxito académico de investigación, colaboración e investigación de los conocimientos; que conlleva al hecho de fortalecer las capacidades de los estudiantes y profesores que manejan la educación STEM, pues tal como lo evidenció el mencionado autor, los efectos alcanzan un mejor aprendizaje académico en la resolución de problemas y las habilidades de pensamiento crítico.

En el mismo orden de ideas, Won (2016), tituló su estudio: "The Wheel Model of STEAM. Education Based on Traditional Korean Scientific Contents, presentada en Incheon National University, Republic of Korea. De allí, surgió el reconocimiento de una educación convergente del talento humano, no obstante, hay deficiencias asociadas con las competencias curriculares, en tanto excluyen a las Humanidades.

Se trató de un diseño de campo, con la aplicación del modelo educativo STEAM agregando el área de las Humanidades (historia, geografía y bibliografía) a las cinco áreas de STEAM (ciencia, tecnología, ingeniería, arte y matemáticas). El principio básico de la educación STEAM se focalizó a utilizar las ciencias tradicionales como medio que se conectan en este enfoque desde el área de las Humanidades. Fue diseñado para incorporar las ocho asignaturas principales como estudios de disciplina de ocho asignaturas e implementarse a través del modelo de rueda, desarrollándose en un sistema multidisciplinar. El modelo educativo STEAM se aplicó en dos escuelas secundarias durante un período de dos años como programas piloto. Ambos casos fueron verificados por la academia e informados en sus beneficios a través del Sistema de Radiodifusión de Corea (KBS). Como resultados se evidenció a través de sesiones de discusión, la participación de los estudiantes en las disciplinas con el intercambio, presentación y discusión de sus productos. Se realizaron escritos de logros de investigación individuales, los estudiantes criticaron el conocimiento existente en la reducción de la construcción.

El estudio concluyó que el modelo educativo STEAM puede ser realizado a través de la ejecución de proyectos, al hacer hincapié en el hecho que los estudiantes deben planificar sus actividades para la resolución de problemas. El aprendizaje basado en proyectos es un método para que los discentes aprendan a través de proyectos, descubran y resuelvan situaciones por sí mismos, lo cual implica una mayor preparación activa y participación en el protagonismo educativo. Resalta el hecho contributivo de la metodología STEAM para que el estudiante se 
convierte en un sujeto activo del proceso de aprendizaje desde la colaboración y cooperación en la solución de los problemas. Por tanto, es aprovechable esta situación didáctica al profundizar en el papel pedagógico que se dinamiza desde el estudiante con la metodología STEAM

Esta investigación previa aporta elementos conceptuales y operativos de interés en la aplicabilidad del enfoque STEAM, como estrategia integral que permite potenciar la formación y especificidad creativa en las competencias de los profesores, puesto que el ámbito de su desarrollo pedagógico en las buenas prácticas atribuidas de manera convergente, desarrollan el éxito educativo para armonizar la cosmovisión integral de las distintas áreas disciplinares y las humanidades en particular, en la propuesta de los proyectos, contenidos científicos e interacción real con los problemas circundantes de la comunidad. El modelo proporciona soportes para profesores e investigadores en el marco de descubrir y desarrollar energía pedagógica aplicable a diversidad de contenidos educativos.

Igualmente, Ruiz (2017), tituló su tesis doctoral: "Diseño de Proyectos STEAM a Partir del Currículum Actual de Educación Primaria Utilizando Aprendizaje Basado en Problemas, Aprendizaje Cooperativo, Flipped Classroom y Robótica Educativa" presentada en la Universidad CEU Cardenal Herrera, València, España. El objetivo general fue diseñado un proyecto de aprendizaje STEAM para alumnos de $4^{\circ}, 5^{\circ}$ y $6^{\circ}$ de educación primaria en el que, a través del uso de robótica educativa como herramienta, se introduzcan metodología de aprendizaje basado en problemas, aprendizaje cooperativo y sesiones de flipped classroom.

La investigación se desarrolló con una metodología descriptiva y experimental, con el análisis STEAM del currículum de $4^{\circ}, 5^{\circ}$ y $6^{\circ}$ de educación primaria, el diseño de la propuesta de intervención con estudio piloto y luego, la aplicación de la propuesta, la cual fue llevada a cabo en la Comunidad Valenciana. El análisis se fragmentó en la etapa del aprendizaje STEAM en el currículum con la búsqueda de cualidades o recomendaciones propias del enfoque; análisis de contenidos con el fin de detectar las redundancias curriculares, agrupación de los contenidos en elementos curriculares por similitud y clasificación según disciplinas. Consideró, además, las oportunidades con la definición y propiedades de áreas correspondientes, la búsqueda de los elementos curriculares según disciplina STEAM, fijando las relaciones intra e interdisciplinares y finalmente, la etapa de creación de un mapa de relaciones intererdisciplinarias entre las oportunidades y el resto de las áreas.

Con respecto a los resultados del estudio piloto, los expertos opinaron que la prueba tiene mucha capacidad, de manera global, para detectar estudiantes que saben lo que es la sostenibilidad y los que no lo saben, todos consideraron muy adecuados los aspectos presentados. Ello permitió establecer relaciones con los resultados de la prueba final del proyecto, la plantilla de evaluación de la propuesta y los resultados de la aplicabilidad respecto a la competencia en sostenibilidad. En cuanto a los resultados del sistema de evaluación incorporado dentro del proyecto, algunos docentes apreciaron cierto desvío en el número de 
sesiones, con alcances significativos para trabajar los conceptos de sostenibilidad respecto a la integración curricular. La multi-disciplinariedad implementada con la matemática, artística, tecnología y ciencias, se situó favorable en la apreciación del rendimiento académico.

Entre las conclusiones destaca que la propuesta diseñada es un proyecto cuya estructura metodológica estuvo focalizada en las teorías del aprendizaje y una temática efectiva hacia el proceso de investigación y resolución de problemas. El proceso de diseño y puesta en práctica evidenció que los proyectos de aprendizaje STEAM derivan competencias clave trabajadas. Asimismo, dio cuenta de la incorporación de contenidos y estándares de aprendizaje propios de cada una de las disciplinas STEAM, que, en este caso, tuvo lugar en el proyecto de la sostenibilidad enmarcada dentro del ámbito científico.

Recomendó el enfoque implementado para favorecer el aprendizaje STEAM al garantizar la aparición de los componentes tecnología (T), y artística (A), y dejar abiertas las posibilidades de nuevos diseños, representando así, una herramienta de aprendizaje y no un fin en sí mismo, porque mediante la incorporación de la tecnología permite ser personalizada según los criterios didácticos, pedagógicos y de creatividad del docente y no al contrario, de modo que se respetan la fase de construcción, programación y manipulación de la robótica educativa activa.

Desde el punto de vista metodológico del enfoque STEAM, este estudio contribuye significativamente a valorar la propuesta de integración interdisciplinaria de los contenidos mediante proyectos de aprendizaje basados en problemas, para poner en práctica la creatividad y capacidad del docente al introducir componentes, elementos y situaciones cognitivas e inteligibles de creatividad, innovación y transformación hacia buenas prácticas en la búsqueda de soluciones. Así, el desarrollo curricular ofrece oportunidades novedosas que fundamentan la viabilidad de los proyectos educativos en las unidades curriculares integradas desde diversos contenidos y áreas del conocimiento.

Asimismo, Ozkan \& Topsakal (2017), indagaron acerca de "Examining Students: Opinions about STEAM Activities” en la Yildiz Technical University, Istanbul, Turkey. El propósito de este estudio fue determinar las opiniones de los estudiantes sobre las actividades de STEAM. Metodológicamente, se ubicó en el paradigma cualitativo, al trabajar con estudiantes de $7^{\circ}$ grado $(n=37)$ de una escuela pública en Estambul. Fue utilizado un muestreo intencional. Se aplicaron nueve (9) actividades de STEAM mientras se enseñaba la unidad de Fuerza y Energía. Al finalizar el proceso de enseñanza se consideró la búsqueda de los datos a través de un formulario de evaluación compuesto por preguntas abiertas. Las respuestas proporcionadas se analizaron cualitativamente para determinar las opiniones de estudiantes sobre las actividades. Se implementó el análisis de contenido para Evaluar los datos obtenidos.

Los hallazgos revelaron que los estudiantes mantienen una actitud positiva sobre las actividades STEAM. Se concluyó sobre las buenas prácticas de participación en el aprendizaje e integración de las disciplinas que se les atribuyen a las actividades STEAM, cuestión que genera muy pocas 
opiniones negativas acerca de esta metodología, según la percepción de los estudiantes. Esta condición es significativa en la educación de este tipo, en el alcance de los objetivos de orientación y prosecución de estudios universitarios pues aumenta el número de estudiantes que preferirán profesiones en estas disciplinas. Además, permite que los alumnos encuentren soluciones creativas a los contextos reales donde se identifican problemas de la vida que pueden ser abordadas con la integración de disciplinas que aumentan los conocimientos básicos en ciencias, tecnología, ingeniería, arte y matemáticas.

Desde el punto de vista de los aportes de este estudio previo, se destaca el enfoque de aprendizaje que busca el mayor protagonismo de los estudiantes, pues la metodología STEAM, favorece el dominio de las competencias transversales y permite conectar las prácticas de aula con la realidad social del contexto, generando la participación de diversos agentes sociales en el desarrollo educativo para dar vida pedagógica a los espacios de aprendizaje con el uso de la tecnología disponible en la resolución de problemas reales en cualquier área del conocimiento, pues la dinámica formativa se desarrolla de manera interdisciplinaria.

\section{Fundamentos Teóricos}

\section{Educación STEAM}

El enfoque pedagógico STEAM, es singularmente importante desde el punto de vista estratégico para el desarrollo de las competencias digitales y el conocimiento sostenido en todas las disciplinas del saber científico-académico, lo cual atrae a su aplicabilidad educativa, por el impacto en las diferentes formas de abordar la realidad con la participación activa de los actores multi-disciplinares, que se integran y amplían las oportunidades de mejora en lo social, económico, cultural y formativo como beneficio que aporta nuevos elementos, escenarios, recursos, talentos del equipo humano y competencias, destinadas al foco de atención a los problemas inherentes al contexto real, dinámico y cooperativo de los aprendizajes que puede adaptarse a todos los niveles y modalidades educativas. En este orden de ideas, señala Mendiola (2018), la importancia de la aplicabilidad de este modelo dinámico de la educación que añade a las disciplinas científicas (ciencia, tecnología, ingeniería, matemáticas) además de las humanidades (artes, diseño) valor agregado, pues despliegan la creatividad, interdisciplinariedad y asume la intuición para la realización de proyectos artísticos, científicos y nuevos descubrimientos de expresión personal cargados de imaginación.

A mi entender, la complementariedad de posibilidades que connota este modelo educativo STEAM, de carácter sinérgico, holístico y basado en criterios flexibles, ajustables en el modo combinado en lo científico, artístico, humanístico y tecnológico, cobra vigencia cada vez más, ante la demanda de su promoción en los diversos niveles y modalidades educativas, pues su singularidad potencia las habilidades sociales en la resolución de problemas que incentivan las actuaciones del docente en su campo profesional, para crear actividades y estrategias digitales en el marco de la cultura tecnológica y los proyectos educativos que permitan superar las 
barreras de la fragmentación del conocimiento en el abordaje a los problemas de su competencia.

Es por ello, que el desarrollo dinámico transdisciplinario en el perfil de competencias adquiridas por los actores del hecho educativo, particularmente cuando se involucran en la implementación creativa de estrategias de esta metodología educativa de manera práctica han de asomar alternativas múltiples en la resolución de los problemas, lo cual exige una comprehensión de la realidad misma y sus elementos integrados, ante la red de significados que se conectan de manera compleja en el alcance de los aprendizajes relacionados con los contenidos STEAM, como puntos neurálgicos que avanzan en las observaciones del tercero incluido, situación que va más allá de la mirada simple de la cognición conceptual unidisciplinar y del comportamiento humano identificado en los ámbitos del aprendizaje.

De la misma manera, la experiencia del modelo pedagógico STEAM favorece la investigación en la Universidad, tanto de los estudiantes como de los profesores hacia aporte de nuevos aprendizajes que tienen interés desde acuerdos en pro del éxito de las actividades, actitudes y herramientas tecnológicas implementadas, dan el cumplimiento eficaz desde la manifestación de los proyectos y procedimientos matemáticos, al indagar en nuevos escenarios de descubrimientos creativos, cognitivos y de habilidades, competencias, diseño de productos y aprendizajes que favorecen el uso de las tecnologías de aprendizaje y el conocimiento. Ante esta ejercitación de procesos apropiados de conceptos matemáticos, señalan Giraldo, Molina y Córdoba (2018) la idea de poner en práctica habilidades cognitivas como el razonamiento y la creatividad, a fin de despertar nuevos comportamientos, actitudes y motivaciones positivas en las propuestas de trabajo colaborativo, ante la gama de proyectos de aula que integran áreas de ciencias naturales (biología, física y química), al poner a la disposición de los fenómenos estudiados expresiones matemáticas y la representatividad de planos, arquitectura, gráficos y construcciones creativas, entre otras.

Esta sindéresis de elementos, factores y condiciones hace pensar que los criterios para resolver los problemas y las habilidades en el desarrollo de las capacidades del equipo interceptan la interpretación acerca de la superación que puede existir como barreras epistemológicas entre las disciplinas del saber hacer de la educación. Tal como lo mencionan Carballo y Portero (2018), en el contexto de las asignaturas universitarias y preuniversitarias implicadas en el desarrollo de la ciencia, tecnología, ingeniería, arte y matemática (STEAM) llegando de esta manera a confluir acerca del meta-análisis más amplio y completo para abordar la realidad de los aprendizajes.

Por tanto, el uso de metodologías activas mejora de forma significativa los resultados académicos, lo cual añade valor por encima del uso de clases magistrales, pues se aprende haciendo, desde la práctica pedagógica integral donde se trabaja en diferentes contenidos curriculares. La siguiente figura, concreta los elementos representativos de este tipo de educación. 
Gráfico 1. Perfiles de Interés en la Educación STEAM.

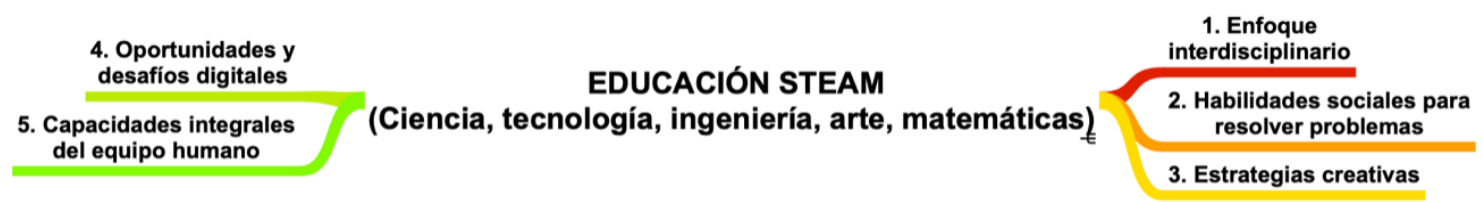

Fuente: Elaboración Propia

La situación que se observa en el gráfico 1, distingue el perfil característico del enfoque interdisciplinario de la educación STEAM, que de acuerdo con Stentoft (2017) es parte asociada al enfoque del aprendizaje basado en problemas, en cuanto a práctica pedagógica potencialmente convincente adecuado para la educación universitaria. Pienso que esta forma de avizorar la realidad circundante de los escenarios sociales, políticos, económicos, culturales y formativos, dan cabida a esa prioridad en la comprensión del desarrollo curricular, más allá de los hechos y contenidos teóricos explícitos en el aula, para llevarlos a la disposición de las capacidades dispuestas por el equipo al valorar el potencial de esta fuerza sinérgica en el tratamiento de los casos pedagógicos de acción recíproca.

Es por ello que Taylor (2016), caracteriza este tipo de metodología STEAM de utilidad para desarrollar habilidades de orden superior de los estudiantes y profesores en las dimensiones del conocimiento y de cara a la indagación de la ciencia como esfuerzo humano añadido, para desplegar escenarios de participación que privilegian la comprensión de sus contenidos, el desarrollo curricular efectivo, innovador y el pensar inteligente y creativamente en la resolución de problemas con el uso de las habilidades tecnológicas. Así, las manifestaciones de sus enfoques y enriquecimiento en el ámbito de la educación, como filosofía curricular que capacita a los profesores de ciencias e involucra los maestros en el desarrollo de la visión humanística, proporciona un espacio de diseño creativo para colaborar de manera integrada con la cosmovisión de un profesor innovador.

Asimismo, el gráfico 1 distingue el perfil relacionado con las oportunidades y desafíos digitales, que hace ver una cosmovisión de los actores sociales inmersos en la dinámica universitaria para entenderse en esa comunicación abierta al mundo globalizado. Por ello, resaltan los razonamientos de Zimmerman (2016) frente a los desafíos de las prácticas que se exploran en la necesidad de mayor confianza desde las capacidades del docente para orientar los eventos y contenidos disciplinares de su especialidad en el marco de acciones educativas asimiladas en torno a la indagación científica o la integración artística a través de implicaciones para el desarrollo de la educación STEAM, para lo cual se han de alcanzar los diversos peldaños de ese conocimiento como soporte de eficiencia real. 
En cuanto a las estrategias creativas que se vinculan al desarrollo de los contenidos y el planteamiento de los proyectos educativos del talento humano incorporados al equipo de la educación STEAM, tal como se observa en el gráfico 1, este perfil artístico es manifestado por Segarra, Natalizio, Falkenberg, Pulford \& Holmes (2018) para mantener al talento humano en las instituciones públicas en tanto la demanda de la fuerza laboral está creciendo y es reconocida por instituciones académicas, sin fines de lucro y gubernamentales, pues ello amenaza la capacidad para reclutar, capacitar y retener de manera efectiva la innovación, por lo tanto, "los educadores científicos están enfrentando algunos de estos desafíos al infundir la creatividad, por medio de las artes, en la educación y la capacitación de los futuros científicos” (p. 1). De modo que la integración de las artes y la ciencia, generan diagramas para saber comunicar la ciencia de manera efectiva, así como la confluencia de apoyo que se devela en la educación y la formación científica. A mi entender, el arte es un puente de comunicación sensible ampliado al conocimiento científico y al potencial cultural que atrae nuevos escenarios de coloridos y espacios de dominio que ayuda a la presentación y representatividad de los significados atribuidos en la diversidad de pensamientos integrados.

Asimismo, la educación STEAM, tal como se avizora en el gráfico 1, activa las habilidades sociales para resolver problemas, cuestión que reafirma lo mencionado por Erwin (2017), en la dinámica hábil que se ha de reafirmar en la formación de los estudiantes, para que asuman las actitudes y conocimientos necesarios para resolver problemas, recopilar y analizar evidencias, integrado a los esfuerzos compartidos con el equipo en la planificación y ejecución de los proyectos saludables. A ello se agrega de acuerdo con el autor citado, la determinación de experiencias apropiadas de aprendizaje en el modelo STEAM, cuestión que comparto al reconocer las opciones activas que proyecta este enfoque en la realidad de vivencias y experiencias orientadoras de las actividades educativas específicas de cada una de las áreas que se integran en el hacer, sentir y pensar en los contenidos asequibles del conocimiento.

En cuanto a las capacidades integrales del equipo humano que distingue el gráfico 1, puede decirse en correspondencia con los argumentos de Kelton \& Saraniero (2018), que ante las tensiones significativas de los desempeños en equipo, la educación STEAM promueve colaboraciones para combinar disciplinas como el arte y las matemáticas, como una formas inteligible de superar las barreras multireferenciales que impactan la generación y promoción colaborativa de los aprendizajes catalizando el crecimiento profesional compartido. Cuestión con la cual estoy de acuerdo para poder avanzar ante la idea que retro-actúa en la generación del conocimiento al enfrentar obstáculos de tiempo, recursos, espacios de encuentros con los otros e infraestructuras.

\section{Sociedad del Conocimiento}

La dinámica de los movimientos sociales, conformada por la diversidad de información y comunicación en las instituciones educativas universitarias experimenta un sistema de reformas necesarias para activar la forma de administrar conceptos y desarrollar el currículo en la 
integración de las disciplinas en un ámbito productivo más allá del aula ante el avance de nuevas realidades sensibles de capacidades, habilidades y competencias del talento humano para poder hacer efectivo los eventos que se significan en la sociedad del conocimiento y las maneras de trabajar en equipos colaboradores integrados a las buenas prácticas de formación a fin de contribuir con el desarrollo de las comunidades y del país. Es así como el conocimiento en línea tiene esa condición potencial de facilitar el acceso al mundo globalizado de la información y el conocimiento. En estas innovadoras formas de comunicarse en red, hacen ver Valerio y Herrera (2017), los niveles de organización cultural, económica, educativa y política que se cumplen en el entorno digitalizado de características tecnosocial ante lo cual se despliega el potencial en la generación del conocimiento y la producción científica a gran velocidad.

Estas consideraciones dan cabida al refuerzo y complementariedad de equipos humanos universitarios y escenarios estructurales, técnicos y tecnológicos que revisten interés en la asimilación de los referentes de transformaciones globales del orden internacional, ante la idea creativa globalizada de progreso que se ha venido gestando en el ámbito educativo a condicionar la innovación y modernización curricular tomando como base la plataforma tecnológica para generar y difundir el conocimiento científico que marca pautas de responsabilidad organizacional para el avance de los modelos pedagógicos interdisciplinares en función de involucrarse en la sociedad del conocimiento.

Tal situación es representativa de los cambios y el sistema de reformas que ataren a los universitarios, tal como lo afirman Valdés y Gutiérrez (2018), pues merece reflexión acerca del sentido educativo y la detección de las prioridades pedagógicas de los actores universitarios, frente a los retos planteados de las competencias digitales y la integración de aspectos relativos a la inteligencia emocional, ciudadanía, valores, lo que significa, de acuerdo con mi percepción que las mismas habilidades sociales de comunicación e información están presentes como categorías inherentes a los nuevos modelos pedagógicos en la sociedad del conocimiento para la formación.

En este sentido, considero que el escenario universitario se ofrece como ámbito ideal para poder enfatizar su funcionamiento adaptable, flexible, sensibilizados ante la digitalización del conocimiento en la formación de profesionales con capacidad científica y tecnológica, aplicable en la generación innovadora de abordar los problemas de la vida práctica, integración de saberes y ejercicio ciudadano colaborativo en red ante la emergencia de una realidad que exige su tratamiento intelectivo de los profesionales universitarios mediante procedimientos innovadores, trascendentes y multidisciplinarios dentro del espiral creciente de información multisectorial.

En palabras de Karpov (2016), la sociedad del conocimiento se muestra como génesis de de nuevas cualidades sociales y educativas que se formula para un trabajador industrial en la idea de entrenamiento avanzado formado en la universidad, bajo los principios de la 
investigación, continuidad científica y cognitiva relacionadas con sus competencias dinámicas, el aprendizaje creativo y la eficiencia académica, dada la preparación cognitiva especializada.

\section{Universidad: Ámbito Activador de las Competencias STEAM}

Las universidades se muestran como escenarios formativos ideales para asimilar las tecnologías de la información y comunicación y ampliar las posibilidades que éstas ofrecen en la concreción educativa de las competencias digitales, como referencia que privilegia la gestión del conocimiento, el aprendizaje integral, el manejo de tecnologías instrumentales para la innovación y transdisciplinariedad del conocimiento aplicativo a los proyectos dentro de una cultura organizacional que mantenga la dualidad de generar productos e insumos para el despliegue de soluciones a los problemas de la realidad social, política, económica, cultural y educacional. En esta perspectiva, Maderick, Zhang \& Hartley (2015), hacen referencia a la autoevaluación subjetiva y evaluación objetiva en siete dominios de competencia digital para profesores en formación al punto de confirmar el poco dominio de esta habilidad de los profesores en formación. Por tanto, se requiere mejorar esta situación útil en prácticas que ayudan a ajustar sus percepciones y actitudes con respecto a la tecnología pues ofrece oportunidades la capacitación en esta condición.

Ante esta realidad, la renovación, innovación y modernización de la plataforma tecnológica de las universidades ha de dar pasos adelantados hacia la adquisición de estos equipos que atraigan la dinámica formativa ante la presencia de mayor dominio por parte de los profesores a fin de significar estas habilidades en el desarrollo de los cursos on line y proporcionar las nuevas oportunidades a sus estudiantes en la conformación de procesos y actividades diseñadas en este marco de la educación STEAM, transformadora de los entornos virtuales tridimensionales (3D) para asimilar el devenir de la era digital en la educación que se adelanta hacia el futuro.

Tal como lo mencionan Steve, Cela \& Gisber (2016), se trata de la competencia digital colaborativa ETeach $3 D$, que se ha construido utilizando el enfoque de investigación de diseño educativo. Se fundamenta en ciclos iterativos sucesivos en criterios de utilidad, validez y efectividad que integra varias estrategias y niveles de complejidad para mejorar la capacitación, digitalización y evaluación del dominio curricular. De allí que, esta caracterización universitaria dependiente y localizada en lo que ha de ser un docente innovador en la era digital, ha de revestir importancia en la consideración de sus funciones que precisan el incremento de la gestión del conocimiento y la búsqueda de mayor productividad, pues considero que las implicaciones del desempeño universitario avizoran el quehacer alternativo tecnológico de los desempeños efectivos convergentes con los distintos roles que exhiben en su legitimación como actores con el compromiso organizacional y la responsabilidad social en la divulgación de nuevos conocimientos tecnológicos ajustados al incremento de la productividad social.

\section{Retos Tecnológicos en la Universidad Actual: Perspectiva de Educación STEAM}

La universidad como foco entendible institucional que avanza en la creación del conocimiento se ve influenciada por los eventos que explica la formación de profesionales en el marco de los 
aprendizajes tecnológicos en marcha hacia la construcción de una cultura innovadora en red de saberes, que ha de incrementar la productividad del trabajo docente, de investigación y extensión más allá de la dinámica integral de las disciplinas en el trasfondo de sembrar nuevos incentivos en el uso efectivo de los equipos tecnológicos para introducir correcciones y un sistema de mejoras en el contacto con las diversas comunidades en las implicaciones del conocimiento sensible.

Desde este contexto, se afianzan los razonamientos de Ozkan \& Topsakal (ob. cit.), en el marco de las disciplinas que se integran en la educación STEAM, ante la necesidad de comprender la realidad de los aprendizajes universitarios, en este caso; como una versión totalizante, holística, no fragmentada en las dimensiones, criterios, principios y atributos de los fenómenos, para la generación del conocimiento. Esta metodología se basa en educación STEM, la cual se derivó ante circunstancias que exigían añadir los componentes del arte y el diseño, según el Ministerio de Educación, Ciencia y Tecnología de España, a partir del 2011, que ahora agrega esta disciplina artística, al acrónimo inglés de la Ciencia, Tecnología, Ingeniería, Arte y Matemáticas. Su propósito se destina a mejorar las habilidades y capacidades de los actores educativos a la resolución de problemas además de impactar la motivación hacia el interés por la ciencia y tecnología, adaptable a los escenarios educativos en cualquier nivel y tipo.

En este sentido, refieren Maddena, Baxtera, Beauchampa, Boucharda, Habermasa \& Huff (2013) las experiencias educativas de programas multidisciplinarios destinados a fomentar el pensamiento creativo, donde se distingue el foco de atención en la combinación de estudios en las artes, humanidades y campos STEM, como escenarios de participación e innovadora en el arte, ciencias naturales y sociales, ciencias de la computación y matemáticas, lo cual añade valor a la multireferencialidad de hechos y alternativas para desarrollo el abordaje de los proyectos versátiles tecnológicos, desde la conformación de equipo de profesores STEAM con capacidades creativas que examinan los contextos industriales, de negocios, empresariales y de la educación para la modernización, transformación e innovación modélica educativa de los proyectos de investigación para abordar los complejos problemas que enfrenta la sociedad humana.

Las consideraciones antes expuestas, han de permiten abordar algunos aspectos reflexivos derivados de la dinámica de integración y potenciación tecnológica de las universidades en Ecuador, para entrar a la era de la digitalización del conocimiento. Esta condición en palabras de Soriano (2015), son indispensables en la comunidad de aprendizajes para que los estudiantes puedan participar, colaborar y mantenerse en contacto a través de la red, interactuando en la construcción del conocimiento, lo cual es fundamental para la innovación como práctica académica sensible al mundo de la era digital, según entiendo dentro de una cultura tecnológica que refleja avances, desarrollo y potencial de comunicación más allá del aula, hacia escenarios de la comunidad global promotores del nuevo modo de trabajo de niveles virtuales. Considero que la universidad debe revisar y reimpulsar estos eventos como una función rectora para el desarrollo de la investigación, docencia y extensión, dentro de los principios de la democratización del conocimiento. 
Al respecto señalan Feyen, Milia, Van Hoof, Quinde, Ochoa, Abril \& Bojorque (2016), que la contextualización de la productividad universitaria en el Ecuador, en términos de los retos tecnológicos afrontados para la formación y los esfuerzos de publicación similares en Sudamérica y Europa occidental, en tanto; a los efectos de las causas de la escasez de investigación y publicación en Ecuador sugieren la necesidad de acogerse a las estrategias correctivas que se centren en ajustar el perfil y las actividades de las Instituciones de Educación Superior (IES) de Ecuador para saltar la brecha, los retos y las implicaciones de la búsqueda concreta de las competencias digitales de este siglo XXI; impulsada por la innovación y el conocimiento. Pienso que la plataforma tecnológica necesaria para solidificar y superar estas expectativas, tiene su sustento en la proliferación de mayores estímulos, apoyos gubernamentales y seguimiento a los programas que establezcan estas iniciativas revisadas por el Estado ante la idea de la investigación tecnológica y cristalización de la educación STEAM en el servicio integrado a los nuevos aprendizajes que exige la modernización de la educación universitaria en el Ecuador para colocarse a la par de las universidades internacionales.

\section{Conclusión.}

Como espacio reflexivo, acoto el hecho de ir preparando para la sociedad del conocimiento en los escenarios humanos, sociales, políticos, culturales y educacionales a través de la educación STEAM, como parte fundamental de la creatividad, al divisar las estructuras actuales de la institucionalidad y las competencias directivas que abren paso en la información global al designar como un hecho, la mencionada metodología, examinar los diagnósticos en la realidad circundante, el conjunto de actuaciones requeridas de carácter interdisciplinarias, en tanto surja el liderazgo científico, tecnológico, de ingeniería, artes y en las matemáticas para develar soluciones globales a la realidad de los problemas que emergen, en diversidad de circunstancias activantes del pensamiento complejo en el núcleo de las transformaciones curriculares necesarias de activar. Además, de elaborar programas sólidos que se centran en ofrecer a los estudiantes, la oportunidad de participar en actividades significativas, dentro de lo que ha de ser una investigación científica auténtica, transdisciplinaria y compleja en el sentido del aprendizaje humano.

\section{Referencias Bibliograficas}

1. Carballo, A y Portero, M. (2018). 10 ideas clave. Neurociencia y educación. Aportaciones para el aula. Barcelona: GRAÓ.

2. Erwin, H. (2017). Full STEAM ahead in physical education. Journal of Physical Education, Recreation \& Dance. 88 (1), 3-4. Recuperado en: https://www.tandfonline.com/doi/full/10.1080/07303084.2016.1249759, https://doi.org/10.1080/07303084.2016.1249759.

3. Feyen, J., Milia, M., Van Hoof, H., Quinde, K., Ochoa, V., abril, V \& Bojorque, J. (2016). Improving the publication visibility of Ecuador's higher education system. MASKANA, 7 (2), 113-125. Recuperado en: http://dspace.ucuenca.edu.ec/bitstream/123456789/26256/3/MASKANA\%207208.pdf, https://doi.org/10.18537/mskn.007.002.08. 
4. Giraldo, F., Molina, J y Córdoba, F. (2018). (Compls.). Experiencias de investigación en escenarios escolares. Elementos para una transformación educativa y social. Medellín: Instituto Tecnológico Metropolitano.

5. Jho, H., Hong, O \& Song, J. (2016). An analysis of stem/steam teacher education in korea with a case study of two schools from a community of practice perspective. EURASIA Journal of Mathematics, Science and Technology Education. 12 (7), 1843-1862. Recuperado en: http://www.ejmste.com/An-Analysis-of-STEM-STEAM-Teacher-Education-in-Koreawith-a-Case-Study-of-Two-Schools-from-a-Community-of-Practice-

Perspective,61321,0,2.html, DOI: https://doi.org/10.12973/eurasia.2016.1538a

6. Karpov, A. (2016). Education in the knowledge society: genesis of concept and reality. International Journal of Environmental \& Science Education. 11 (17), 9949-9958. Recuperado en: https://journals.sagepub.com/doi/full/10.1177/0047239516661713, file://C:/Documents\%20and\%20Settings/Usuario/Mis\%20documentos/Downloads/IJESE 1220article582d8da90493f.pdf

7. Kelton, M \& Saraniero, P. (2018). STEAM y partnerships: a case of interdisciplinary professional development and collaboration. Journal of Museum Education. 43 (1) 55-65. Recuperado

en: https://www.tandfonline.com/doi/abs/10.1080/10598650.2017.1419772?src=recsys\&journ alCode=rjme20, DOI: 10.1080/10598650.2017.1419772

8. Ruiz, F. (2017). Diseño de proyectos STEAM a partir del currículum actual de educación primaria utilizando aprendizaje basado en problemas, aprendizaje cooperativo, Flipped Classroom y robótica educativa. Tesis doctoral no publicada. Universidad CEU Cardenal Herrera, València, España. Recuperado en: http://dspace.ceu.es/handle/10637/8739

9. Maddena, M., Baxtera, M., Beauchampa, H., Boucharda, K., Habermasa, D \& Huff, M. (2013). Rethinking STEM education: an interdisciplinary steam curriculum. Procedia Computer Science. 20 (1), 541-546. doi: 10.1016/j.procs.2013.09.316. Recuperado en: file://C:/Documents\%20and\%20Settings/Usuario/Mis\%20documentos/Downloads/rethink ing-stem-education-an-interdisciplinary-steam-curriculum.pdf.

10. Maderick, J., Zhang, S \& Hartley, K. (2015). Preservice teachers and self-assessing digital competence. Journal Education Computing Research. 54 (3), 326-351. Recuperado en: https://journals.sagepub.com/doi/abs/10.1177/0735633115620432?journalCode=jeca. https://doi.org/10.1177/0735633115620432

11. Mendiola, M. (2018). Revisión de escenarios digitales de aprendizaje. En: Construcción social de una cultura digital educativas. México: Universidad Pedagógica Nacional/Universidad Autónoma Metropolitana/SOMESE. 5-18.

12. Ozkan, M., \& Topsakal, U. (2017). Examining students. Opinions about steam activities. Journal of Education and Training Studies. 5 (9), 115-123. E-ISSN 2324-8068. URL: http://jets.redfame.com.Recuperado en: https://files.eric.ed.gov/fulltext/EJ1151682.pdf.

13. Rodríguez, E. (2005). Metodología de la investigación. La creatividad, el rigor del estudio y la integridad son factores que transforman al estudiante en un profesionista de éxito. México: Universidad Juárez Autónoma de Tabasco. 
14. Segarra, V., Natalizio, V., Falkenberg, C., Pulford, S \& Holmes, R. (2018). STEAM: Using the arts to train well-rounded and creative scientists. Journal Microbiology \& Biology Education. 19 (1), 19.1.53. DOI: 10.1128/jmbe.v19i1.1360. Recuperado en: https://www.ncbi.nlm.nih.gov/pmc/articles/PMC5969448/

15. Soriano, E. (2015). (Edit.). Rethinking education for a global, transcultural world. Estados Unidos: Informationg Age Publishing.

16. Stentoft, D. (2017). From saying to doing interdisciplinary learning: Is problem-based learning the answer? Active Learning in Higher Education. 18 (1), 51-61. Recuperado en: https://journals.sagepub.com/doi/full/10.1177/1469787417693510, https://doi.org/10.1177/1469787417693510.

17. Steve, F., Cela, J \& Gisber, M. (2016). ETeach3D: designing a 3d virtual environment for evaluating the digital competence of preservice teachers. Journal of Educational Computing Research. 54 (6), 816-839. Recuperado en: https://journals.sagepub.com/doi/abs/10.1177/0735633116637191. https://DOI.org/10.1177/0735633116637191.

18. Taylor, P. (2016). Why is a STEAM curriculum perspective crucial to the 21 st century? Research Conference. 89-93. Recuperado en: https://research.acer.edu.au/cgi/viewcontent.cgi?article=1299\&context=researchconference 19. Valerio, G y Herrera, D. (2017). Online social networks as a communication channel for open access journals. Revista Latina de Comunicación Social. 72, 1341-1350. Recuperado en: http://www.revistalatinacs.org/072paper/1221/71en.html, DOI: 10.4185/RLCS-2017-1221-71en

20. Valdés, V y Gutiérrez, P. (2018). Las urgencias pedagógicas en la sociedad del aprendizaje y el conocimiento. Un estudio para la reflexión sobre la calidad en el nuevo modelo educativo. Multidisciplinary Journal of Educational Research. (8), 1-28. DOI: 10.17583/remie.2018.3199. Recuperado en : http://dx.doi.org/10.17583/remie.2018.3199.

21. Won O. (2016). The wheel model of steam. education based on traditional Korean scientific contents. Tesis doctoral no publicada. Incheon National University, Republic of Korea. Recuperado de: DOI: https://doi.org/10.12973/eurasia.2016.1263a

22. Yildirim, B. (2016). An examination of the effects of science, technology, engineering, mathematics (STEM) applications and mastery learning integrated into the 7th grade science course. Tesis doctoral no publicada. Gazi University, Ankara.

23. Zimmerman, A. (2016). Developing confidence in steam: exploring the challenges that novice elementary teachers face. The STEAM Journal. 2 (2), 1-11. Recuperado en: https://scholarship.claremont.edu/cgi/viewcontent.cgi?referer=https://www.google.com/\&h ttpsredir $=1 \&$ article $=1122 \&$ context=steam

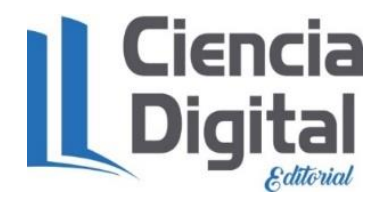




\section{PARA CITAR EL ARTÍCULO INDEXADO.}

Santillán Aguirre, J., Cadena Vaca, V. del C., \& Cadena Vaca, M. (2019). Educación Steam: entrada a la sociedad del conocimiento. Ciencia Digital, 3(3.4.), 212-227. https://doi.org/10.33262/cienciadigital.v3i3.4.847

\section{LCiencia}

El artículo que se publica es de exclusiva responsabilidad de los autores y no necesariamente reflejan el pensamiento de la Revista Ciencia Digital.

El artículo queda en propiedad de la revista y, por tanto, su publicación parcial y/o total en otro medio tiene que ser autorizado por el director de la Revista Ciencia Digital.
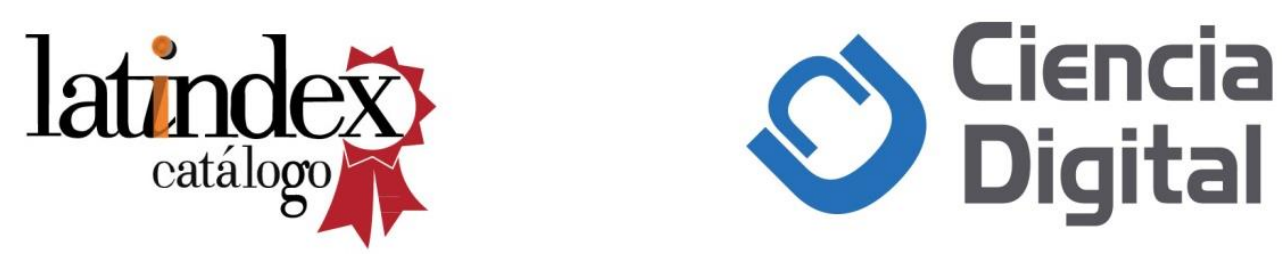